\author{
Andrea CHIRICOZZI ${ }^{1,2, a}$ \\ Antonio COSTANZO ${ }^{3,4, \text { a }}$ \\ Maria Concetta FARGNOLI ${ }^{5}$, a \\ Piergiorgio MALAGOLI ${ }^{6}$, a \\ Stefano PIASERICO ${ }^{7}$, \\ Paolo AMERIO ${ }^{8}$ \\ Giuseppe ARGENZIANO ${ }^{9}$ \\ Nicola BALATO ${ }^{10}$ \\ Federico BARDAZZI ${ }^{11}$ \\ Luca BIANCHI ${ }^{12}$ \\ Carlo Giovanni CARRERA ${ }^{13}$ \\ Andrea CONTI ${ }^{14}$ \\ Paolo DAPAVO $^{15}$ \\ Clara DE SIMONE ${ }^{2,16}$ \\ Francesco LOCONSOLE ${ }^{17}$ \\ Ada LO SCHIAVO 9 \\ Giovanna MALARA ${ }^{18}$ \\ Maria Letizia MUSUMECI ${ }^{19}$ \\ Aurora PARODI ${ }^{20}$ \\ Ketty PERIS ${ }^{2}$ \\ Francesca PRIGNANO ${ }^{21}$ \\ Franco RONGIOLETTI ${ }^{22,23}$ \\ Marina TALAMONTI ${ }^{12}$ \\ Concetta POTENZA ${ }^{24, \text { a }}$
}

${ }^{1}$ Istituto di Dermatologia, Università Cattolica, Largo Francesco Vito, 1, 00168 Rome, Italy

${ }^{2}$ Fondazione Policlinico Universitario A. Gemelli

IRCCS, Largo Agostino Gemelli 8, 00168 Rome, Italy

${ }^{3}$ Dermatology Unit Department of Biomedical

Sciences, Humanitas University, Via Rita Levi

Montalcini, 20089 Pieve Emanuele (MI) Italy

${ }^{4}$ Skin Pathology Laboratory, Humanitas Research

Hospital IRCCS, Via Manzoni 56, 20089 Rozzano,

(MI) Italy

${ }^{5}$ Dermatology, Department of Biotechnological and

Applied Clinical Sciences, University of L'Aquila, Via

Vetoio, Coppito, 67100 L'Aquila, Italy

${ }^{6}$ Head Psocare Unit IRCCS Policlinico San Donato,

Piazza Edmondo Malan, 2, 20097 San Donato,

Milano, Italy

${ }^{7}$ Department of Dermatology, University of Padova,

Via Vincenzo Gallucci, 4, 35121 Padova, Italy

${ }^{8}$ Department of Medicine and Aging Science and

Dermatologic Clinic, University "G. d'Annunzio", Via

dei Vestini, 3166100 Chieti-Pescara, Chieti, Italy

${ }^{9}$ Dermatology Unit, University of Campania Luigi

Vanvitelli, Viale Abramo Lincoln, 5, 81100 Caserta,

Naples, Italy

${ }^{10}$ Department of Dermatology, University of Naples

Federico II, Corso Umberto I, 40, 80138 Naples, Italy

${ }^{11}$ Dermatology, Department of Experimental,

Diagnostic and Specialty Medicine, University of

Bologna, Via Zamboni, 33, 40126 Bologna, Italy

${ }^{12}$ Dermatology Unit, Department of Systems

Medicine, Tor Vergata University of Rome, Facoltà di

Medicina e Chirurgia Via Montpellier 1 00133Italy

${ }^{13}$ Fondazione Cà Granda IRCCS Maggiore Policlinico

Hospital, Via della Commenda, 10, 20122 Milan, Italy

${ }^{14}$ Dermatology Unit, Azienda Ospedaliero

Universitaria di Modena, Largo del Pozzo, 71, 41125

Modena, Italy

${ }^{15}$ ASO City of Health and Science, University

Dermatological Clinic, Corso Vittorio Emanuele II,

62, 10121 Torino Italy

${ }^{16}$ Institute of Dermatology, Catholic University of the

Heart, Largo Francesco Vito, 1, 00168 Rome, Italy

${ }^{17}$ Department of Dermatology, University of Bari,

Piazza Umberto I, 1, 70121 Bari, Italy

${ }^{18}$ UOC of Dermatology, Great Metropolitan Hospital

"BMM", Via Giuseppe Melacrino, 21, 89124 Reggio

Calabria, Italy

a These authors contributed equally

\section{Guselkumab: an anti-IL-23 antibody for the treatment of moderate-to-severe plaque psoriasis}

Guselkumab, a subcutaneously administered fully human IgG1 $\lambda$ monoclonal antibody that selectively inhibits the p19 subunit of interleukin 23 , is approved in both the USA and the EU for the treatment of adult patients with moderate-to-severe plaque psoriasis. The efficacy and safety of guselkumab were demonstrated in four randomized, double-blind, Phase III trials (VOYAGE 1 and 2, NAVIGATE, and ECLIPSE), which demonstrated high levels of clinical response over three years of continuous treatment, regardless of sex, age, body weight, and race, maintaining a favourable safety profile and long-term tolerability. Guselkumab was shown to be efficacious in patients with prior failure of other biologics, including adalimumab and ustekinumab, and was superior to both adalimumab and secukinumab in head-to-head trials. Guselkumab efficacy was also observed in the treatment of psoriasis localized in difficult-to-treat body regions including the scalp, palms and/or soles, and fingernails. Treatment with guselkumab improved health-related quality of life and patient-reported signs and symptoms. Guselkumab has a consistently favourable safety profile and is well tolerated over the long-term. Clinical development of guselkumab as a treatment is ongoing for other immune-mediated inflammatory diseases, including psoriatic arthritis, Crohn's disease, and ulcerative colitis. In the overall management of patients with plaque psoriasis, guselkumab is a robust treatment option with durable maintenance of response over time.

Key words: efficacy, guselkumab, interleukin 23, plaque psoriasis, safety 


\author{
${ }^{19}$ Dermatology Clinic, University of \\ Catania, A.O.U. Policlinico-Vittorio \\ Emanuele, Via S. Sofia 78, 95125 Catania, \\ Italy \\ ${ }^{20}$ DiSSal Section of Dermatology \\ University of Genoa, Policlinic-Hospital \\ San Martino IRCCS, Largo R. Benzi 10 \\ 16132 Genoa, Italy \\ ${ }^{21}$ Unit of Dermatology, Department of \\ Health Science, University of Florence, \\ Piazzale Michelangiolo, 4150125 Florence, \\ Italy \\ ${ }^{22}$ Dermatology Clinic, Department of \\ Medical Sciences and Public Health, \\ University of Cagliari, Via Università 40, \\ 09124 Cagliari, Italy \\ ${ }^{23}$ IRCCS San Raffaele Hospital, Vita Salute \\ University, Milan \\ ${ }^{24}$ Dermatology Unit "Daniele Innocenzi", \\ Department of Medical-Surgical Sciences \\ and Biotechnologies, Sapienza University \\ of Rome, Polo Pontino, Corso della \\ Repubblica, 7904100 Latina, Rome, Italy
}

Reprints: Maria Concetta Fargnoli

<mariaconcetta.fargnoli@univaq.it>

(C) The Author(s) 2021

Article accepted on 01/07/2020

$\mathrm{P}$

soriasis is a chronic inflammatory, immunemediated skin disorder $[1,2]$, estimated to affect approximately $2-4 \%$ of the population in Western countries [3].

The therapeutic armamentarium for the treatment of moderate-to-severe psoriasis includes traditional systemic therapies (methotrexate, cyclosporine, and acitretin) and phototherapy. However, their use may be limited by poor effectiveness, potential long-term cumulative toxicity, or frequent monitoring requirements [4]. The introduction of biologic therapies represented an important step forward in the care and treatment of patients with moderate-to-severe psoriasis, providing long-term control of the disease with an excellent safety profile.

With recent advances in our understanding of the complex pathophysiology of psoriasis, the pro-inflammatory cytokine interleukin 23 (IL-23) has become an important therapeutic target in the development of biologic drugs for psoriasis [5-7].

Guselkumab, a fully human $\operatorname{IgG} 1 \lambda$ monoclonal antibody, is a selective inhibitor of the IL-23p19 subunit. It was the first in its class to be approved by the US Food and Drug Administration (FDA) (13 ${ }^{\text {th }}$ July 2017) and the European Medicines Agency (EMA) (14 ${ }^{\text {th }}$ September 2017) for the treatment of adult patients with moderate-to-severe plaque psoriasis who are eligible for systemic therapy with biologic drugs $[8,9]$. In both the USA and EU, the recommended dosage for the treatment of plaque psoriasis is $100 \mathrm{mg}$ guselkumab, administered by subcutaneous injection at zero and four weeks, followed by a maintenance dose every eight weeks (q8w) thereafter [10-12]. To assure optimal guselkumab dose selection, numerous doses were tested in patients with moderate-to-severe plaque psoriasis in Phase 1 [13] and Phase 2 [14] studies, with the 100-mg q8w dose selected for Phase 3 evaluation. A recent exposure-response modelling analysis, conducted using the Phase 2/Phase 3 data to better understand the dose-response relationship among different guselkumab doses, including untested doses [15], confirmed the selected dose as optimal [16]. The clinical development program of guselkumab included two Phase I trials [13, 17], one Phase II trial (X-PLORE) [14], and four Phase III trials (VOYAGE 1 [18], VOYAGE 2 [19], NAVIGATE [20], and ECLIPSE [21]). Although plaque psoriasis was the first therapeutic indication to be approved, clinical development of guselkumab is ongoing for other immune-mediated inflammatory diseases, including psoriatic arthritis (PsA), Crohn's disease, and ulcerative colitis.

Herein, we provide an overview of guselkumab covering its mechanism of action, pharmacodynamics and pharmacokinetic characteristics, therapeutic efficacy and safety, and provide future perspectives of its potential role in other immune-mediated inflammatory diseases.

\section{Mechanism of action}

IL-23 is predominately expressed by inflammatory dendritic cells and plays a pivotal role in the pathogenesis of chronic autoimmune diseases, particularly psoriasis [22]. IL-23 is a heterodimeric cytokine which, like IL-12, is composed of a unique p19 subunit linked to a p40 subunit, as both belong to the IL-12 cytokine family (figure 1) [23-25]. Currently, the IL-23/IL-17 axis is thought to be the most important pathway in the pathogenesis of psoriasis [26-28]. IL-23 activates the JAK-STAT signalling pathway by binding specifically to the IL-23 receptor, which is located on the surface of target cells, causing the phosphorylation of STAT3 and STAT4 via JAK2 and Tyk2 kinases. This in turn stabilizes T-helper 17 (Th17) cells, which are involved in many immune-inflammatory diseases via their role in the induction of tissue inflammation and tissue destruction $[29,30]$. 


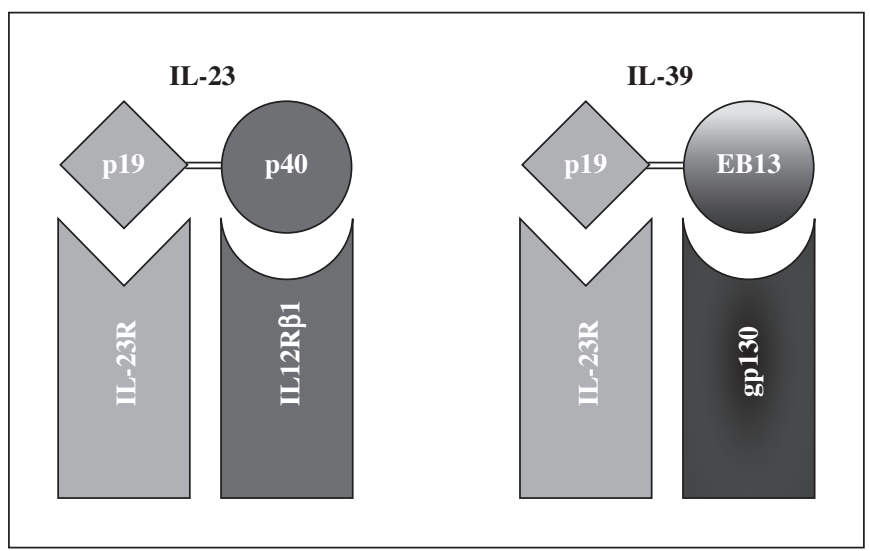

Figure 1. Diagrammatic representation of the heterodimeric cytokine interleukin (IL)-23 (composed of the p19 subunit linked with the p40 subunit) and IL-39 cytokine (composed of the p19 subunit linked with the EB13 subunit), and their receptors.

Several innate immune cells, characterized by expression of the transcription factor retinoic acid receptor-related orphan receptor- $\gamma \mathrm{t}(\mathrm{ROR} \gamma \mathrm{t})$ and collectively known as type 17 cells, respond to IL-23 stimulation. These include Th17 cells, Tc17, IL-17-producing $\gamma \delta \mathrm{T}$ cells, and Group 3 innate lymphoid cells [27, 31]. IL-23 also regulates the differentiation and activation of regulatory $\mathrm{T}$ cells (Tregs). The presence of IL-23 leads to the differentiation of inducible Th17 cells into pathogenic Th17 (expressing ROR $\gamma \mathrm{t}$ ), whereas its absence leads to the development of regulatory Th17 (expressing both ROR $\gamma \mathrm{t}$ and Foxp3 and producing both IL-17 and IL-10) that could play a relevant role in maintaining the mucosal barrier function and in preserving host defence against Candida infections.

\section{Pharmacodynamics and pharmacokinetics}

The guselkumab Phase I proof-of-concept study established that selective antagonism of IL-23 via the inhibition of IL23 was a promising therapeutic approach for the treatment of psoriasis and further supported the central role of the IL-23/IL-17 pathway in the pathogenesis of psoriasis [13]. In this study, an analysis of lesional and non-lesional skin biopsies showed clinical improvement of psoriasis characterized by decreases in epidermal thickness and T-cell and dendritic cell expression in patients with moderateto-severe plaque psoriasis who were treated with a single subcutaneous dose of guselkumab $(10,30,100$, or $300 \mathrm{mg}$ ) compared with placebo. Significant reductions in psoriasis gene expression (both proliferative and inflammatory markers) and serum IL-17A levels were observed in psoriasis patients after 12 weeks of guselkumab therapy [13]. Overall, the data showed that guselkumab specifically regulates expression of IL-17 and IL-17 pathway downstream genes in psoriasis lesions, whereas expression of the IL$12 / \mathrm{T}_{\mathrm{H}} 1$ pathway gene targets remained unchanged during the therapy while still obtaining a clinical response. This demonstrates that the response to guselkumab is not linked to the suppression of IL-12, which is therefore irrelevant from a therapeutic point of view but linked to the selective inhibition of IL-23. However, recent data [32] indicates that another p19-containing cytokine (IL-39) (figure 1) may have pro-inflammatory activities, and at least theoretically can be blocked by guselkumab, raising new hypotheses on the real target of guselkumab in psoriasis.

The pharmacokinetic profiles of guselkumab were shown to be comparable between healthy subjects and patients with psoriasis in a first-in-human, single-ascending-dose study designed to assess the pharmacokinetics, safety, and tolerability of guselkumab [17]. Linear pharmacokinetics of guselkumab, as shown by dose-proportional increases in the mean maximum observed serum concentration $\left(\mathrm{C}_{\max }\right)$ and area under the serum concentration-time curve values, were observed in both populations following intravenous or subcutaneous administration of a single dose ranging from 0.03 to $10 \mathrm{mg} / \mathrm{kg}$ or 10 to $300 \mathrm{mg}$, respectively; mean half-life $\left(\mathrm{T}_{1 / 2}\right)$ values were also consistent between populations (12-19 days for healthy subjects versus 15-17 days for patients with psoriasis) [17].

A population pharmacokinetic model, established using 13,014 pharmacokinetic samples from 1,454 guselkumabtreated patients with moderate-to-severe psoriasis, predicted that it would take between 12 and 14 weeks to achieve steady-state serum guselkumab concentration based on the model-derived elimination $\mathrm{T}_{1 / 2}$ value of 18.1 days [33]. Although body weight was identified as the primary covariate affecting pharmacokinetic variability of guselkumab, dose adjustment based on body weight was not warranted. Neither age, sex, ethnicity, immune response to guselkumab, or concomitant medications had a clinically relevant effect on guselkumab exposure.

The pharmacodynamics and pharmacokinetic properties of guselkumab are shown in table 1.

\section{Therapeutic efficacy in plaque psoriasis}

Guselkumab is effective and safe for the treatment of moderate-to-severe plaque psoriasis according to data from a comprehensive global development program including Phase I-II trials and four Phase III trials (VOYAGE 1 [NCT02207231] [18], VOYAGE 2 [NCT02207244] [19], NAVIGATE [NCT02203032] [20], and ECLIPSE [NCT03090100] [21]).

Clinical response and safety of guselkumab were initially demonstrated in patients with moderate-to-severe plaque psoriasis in a small $(n=24)$ Phase I trial after a single subcutaneous injection of guselkumab at 10,30,100, or $300 \mathrm{mg}$ or placebo [13]. This study supported guselkumab as a promising therapy for psoriasis. In another Phase I, randomized, double-blind, placebo-controlled, single-ascending-dose study $(n=20)$, guselkumab was well-tolerated and exhibited sustained high levels of clinical response in Japanese patients with moderate-to-severe psoriasis [34].

In the Phase II trial X-PLORE (NCT01483599), guselkumab was shown to have a rapid onset of action (efficacy evident by Week 4) with continued efficacy for up to 40 weeks of continuous treatment across a broad range of doses $(5,15,50,100$, and $200 \mathrm{mg})$ and two different dosing intervals (Weeks 0,4 , and $\mathrm{q} 12 \mathrm{w}$ or $\mathrm{q} 8 \mathrm{w}$ ) in 293 patients with moderate-to-severe plaque psoriasis [14]. 
Table 1. Pharmacodynamics and pharmacokinetic properties of guselkumab [10, 13, 17].

\begin{tabular}{|ll|}
\hline Pharmacodynamics & $\begin{array}{l}\text { Reduced expression of IL-23/Th17 pathway genes and psoriasis-associated gene expression profiles } \\
\text { Reduces serum IL-17A, IL-17F and IL-22 levels }\end{array}$ \\
\hline Pharmacokinetics & \\
\hline Absorption & $\begin{array}{l}\text { Mean }( \pm \mathrm{SD}) \text { maximum serum concentration }\left(\mathrm{C}_{\mathrm{max}}\right) \text { of } 8.09 \pm 3.68 \mathrm{mcg} / \mathrm{mL} \text { approximately } 5.5 \mathrm{days} \\
\text { after a single } 100-\mathrm{mg} \mathrm{SC} \text { injection in healthy subjects } \\
\text { Mean }( \pm \mathrm{SD}) \text { steady-state trough serum guselkumab concentrations of } 1.15 \pm 0.73 \mathrm{mcg} / \mathrm{mL} \text { and } \\
1.23 \pm 0.84 \mathrm{mcg} / \mathrm{mL} \text { were demonstrated in two Phase III studies }\end{array}$ \\
\hline Absolute bioavailability & $49 \%$ in healthy subjects after a single 100 -mg subcutaneous injection \\
\hline Volume of distribution & Approximately $7-10 \mathrm{~L}$ \\
\hline Metabolism & $\begin{array}{l}\text { Although the exact metabolic pathway of guselkumab is unknown, its degradation into small peptides } \\
\text { and amino acids via catabolic pathways in a manner similar to endogenous IgG is expected }\end{array}$ \\
\hline Half-life $\left(\mathrm{T}_{1 / 2}\right)$ & $\begin{array}{l}\text { Mean } \mathrm{T}_{1 / 2} \text { approximately } 17 \text { days in healthy subjects and approximately } 15-18 \text { days in patients with } \\
\text { plaque psoriasis }\end{array}$ \\
\hline Elimination & Mean systemic clearance $(\mathrm{CL})$ ranged from 0.288 to $0.479 \mathrm{~L} /$ day across studies \\
\hline
\end{tabular}

IL: interleukin; SD: standard deviation; Th: T-helper.

\section{Phase III trials: VOYAGE 1 and VOYAGE 2 (guselkumab vs placebo vs adalimumab)}

Superiority of guselkumab compared with placebo or adalimumab was demonstrated in two Phase III, multicentre, randomized, double-blind, placebo- and active comparator-controlled (adalimumab) trials; VOYAGE 1 $(n=837)$ [18] and VOYAGE $2(n=992)$ [19]. VOYAGE 1 assessed the outcome of a year of continuous treatment with guselkumab compared with adalimumab. VOYAGE 2 evaluated the efficacy and safety of treatment interruption (to imitate treatment gaps, which occur frequently in clinical practice) and assessed the transition from adalimumab to guselkumab (to replicate switching patients from adalimumab to guselkumab). In both VOYAGE trials, patients were randomized to guselkumab at $100 \mathrm{mg}$ at Weeks 0,4 and $\mathrm{q} 8 \mathrm{w}$, or to placebo or adalimumab; co-primary endpoints were the proportion of patients achieving an Investigator's Global Assessment (IGA) score of cleared/minimal disease (IGA 0/1) and $\geq 90 \%$ improvement from baseline in Psoriasis Area and Severity Index score (PASI 90) at Week 16, comparing guselkumab and placebo groups.

VOYAGE 1 demonstrated superiority of guselkumab compared with placebo and/or adalimumab for all co-primary outcome measures and all major secondary endpoints (table 2). Guselkumab was superior to adalimumab at the Week-16 endpoints of IGA 0/1 and PASI 90 (all $p<0.001)$ and response rates continued to improve beyond Week 16 [18]. Approximately half of all patients treated with guselkumab achieved complete clearance (IGA 0) at Weeks 16, 24, and $48(47.7 \%, 52.6 \%$, and $50.5 \%$, respectively) compared with less than a third of patients receiving adalimumab $(26.3 \%, 29.3 \%$, and $25.7 \%$, respectively). This is noteworthy as total skin clearance has been associated with optimal health-related

Table 2. Coprimary endpoints and major secondary endpoints in VOYAGE 1 and VOYAGE 2.

\begin{tabular}{|c|c|c|c|c|c|c|}
\hline & \multicolumn{3}{|c|}{ VOYAGE 1 [18] } & \multicolumn{3}{|c|}{ VOYAGE 2 [19] } \\
\hline & $\begin{array}{l}\text { PBO } \\
(n=174)\end{array}$ & $\begin{array}{l}\text { Gus } \\
(n=329)\end{array}$ & $\begin{array}{l}\text { Ada } \\
(n=334)\end{array}$ & $\begin{array}{l}\text { PBO } \\
(n=248)\end{array}$ & $\begin{array}{l}\text { Gus } \\
(n=496)\end{array}$ & $\begin{array}{l}\text { Ada } \\
(n=248)\end{array}$ \\
\hline Week 16 & & 3 & & & & \\
\hline PASI 75 & $10(5.7)$ & $00(91.2)^{*}$ & $244(73.1)$ & $20(8.1)$ & $428(86.3)^{*}$ & $170(68.5)$ \\
\hline PASI 90 & $5(2.9)$ & $241(73.3) *$ & $166(49.7)$ & $6(2.4)$ & $347(70.0) *$ & $116(46.8)$ \\
\hline IGA 0/1 & $12(6.9)$ & $280(85.1)^{*}$ & $220(65.9)$ & $21(8.5)$ & 417 (84.1)* & $168(67.7)$ \\
\hline IGA 0 & $2(1.1)$ & 157 (47.7)* & $88(26.3)$ & $2(0.8)$ & 215 (43.3)* & $71(28.6)$ \\
\hline \multicolumn{7}{|l|}{ Week 24} \\
\hline PASI 90 & - & $264(80.2) \dagger$ & $177(53.0)$ & - & $373(75.2) \dagger$ & $136(54.8)$ \\
\hline IGA 0/1 & - & $277(84.2) \dagger$ & $206(61.7)$ & - & $414(83.5) \dagger$ & $161(64.9)$ \\
\hline IGA 0 & - & $173(52.6) \dagger$ & $98(29.3)$ & - & $257(51.8) \dagger$ & $78(31.5)$ \\
\hline \multicolumn{7}{|l|}{ Week 48} \\
\hline PASI 90 & - & $251(76.3) \dagger$ & $160(47.9)$ & - & - & - \\
\hline IGA 0/1 & - & $265(80.5) \dagger$ & $185(55.4)$ & - & - & - \\
\hline IGA 0 & - & $166(50.5) \dagger$ & $86(25.7)$ & - & - & - \\
\hline
\end{tabular}

Ada: adalimumab; Gus: guselkumab; IGA: Investigator Global Assessment; $n$ : number of patients; PASI 75: 75\% or greater improvement from baseline in Psoriasis Area and Severity Index; PASI 90: $90 \%$ or greater improvement from baseline in Psoriasis Area and Severity Index; PBO: placebo.Coprimary endpoints for both VOYAGE 1 and VOYAGE 2 were the proportion of patients achieving IGA 0/1 at Week 16 and the proportion of patients achieving a PASI 90 at Week 16 in the guselkumab group compared with placebo. Values are reported as $n(\%)$. $p<0.001$ for guselkumab vs. placebo. $\dagger p<0.001$ for guselkumab vs. adalimumab. 
quality of life (HRQoL) benefits for patients with psoriasis [35].

VOYAGE 2, which consisted of a placebo-controlled period (Weeks 0-16), an active comparator-controlled period (Weeks 0-28), and a randomized withdrawal and retreatment period for patients who achieved PASI 90 from baseline to Week 28 (Weeks 28-72), demonstrated that guselkumab was highly effective in the treatment of patients with moderate-to-severe psoriasis, thereby confirming the results of VOYAGE 1. In VOYAGE 2, guselkumab demonstrated superiority to placebo and to adalimumab for all co-primary endpoints and major secondary endpoints at Weeks 16 and 24 (all $p<0.001$ ) (table 2) [19].

VOYAGE 2 also demonstrated the benefits of maintenance versus withdrawal therapy and the effectiveness of guselkumab in adalimumab non-responders (defined as $<$ PASI 90 response at Week 24) [19]. For guselkumab Week-28 responders, clinical responses (IGA 0/1 and 0 , and PASI 75, 90, and 100) were maintained by significantly higher proportions of patients who continued to receive guselkumab (maintenance group) compared with patients re-randomized to placebo (withdrawal group) at Week 48 (all $p<0.001$ ). Continuous guselkumab treatment was associated with a better persistence of response, whereas recurrence of psoriasis and a reduction in HRQoL were observed in patients interrupting treatment. VOYAGE 2 also showed that switching to guselkumab at Week 28 was effective in adalimumab non-responders $(n=112)$, with $66.1 \%$ achieving PASI 90 and 28.6\% achieving PASI 100 at Week 48 (relative to baseline). Furthermore, high levels of clinical efficacy were demonstrated at Week 100 following guselkumab treatment in adalimumab PASI 90 non-responders who initiated guselkumab at Week 52 in VOYAGE 1 or at Week 28 in VOYAGE 2 [36]. These results demonstrate the need for continuous therapy to maintain clinical response and show that adalimumab nonresponders can be successfully transitioned to guselkumab. Based on a pooled analysis of VOYAGE 1 and 2 data $(n=1,829)$, guselkumab achieved superior efficacy (IGA $0 / 1$ and IGA 0) over adalimumab at Week 24, which was independent of sex, race, age, disease duration $<15$ years or $\geq 15$ years, baseline PASI $<20$ or $\geq 20$, prior exposure to biologics, the presence of PsA, and body weight $\leq 90 \mathrm{~kg}$ or $>90 \mathrm{~kg}$ [37]. Regardless of baseline weight $(<74.6, \geq 74.6$ to $<86.4$, $\geq 86.4$ to $<100$, and $\geq 100 \mathrm{~kg}$ ), a significantly greater proportion of guselkumab-treated patients achieved consistently high PASI 90 and IGA 0/1 response rates at Week 24 (all $p \leq 0.005$ vs. adalimumab), suggesting that, for guselkumab, bodyweight does not influence response rates [38]. A separate post-hoc analysis of VOYAGE 1 and 2 data identified a rapid clinical response following treatment with guselkumab, with PASI 90 achieved at a median time of $\leq 12$ weeks compared with $\leq 16$ weeks for adalimumabtreated patients, and PASI 100 achieved at $\leq 24$ weeks for guselkumab but not reached for adalimumab-treated patients [39].

Due to the chronic nature of psoriasis, sustained response to treatment is imperative. The long-term extension of VOYAGE 1, in which all patients received open-label guselkumab at the approved dosage regimen of $100 \mathrm{mg}$, administered q8w starting at Week 52, demonstrated durable maintenance of high levels of efficacy for up to three years of continuous treatment, even among adalimumabtreated patients who crossed over to guselkumab [40]. The proportions of patients achieving PASI 90 at Weeks 100 and 156 , respectively, in the guselkumab group were $82.1 \%$ and $82.8 \%$ in VOYAGE 1 and $79.1 \%$ and $77.2 \%$ in VOYAGE 2. Approximately half of all patients at Week 100 and Week 156 achieved complete clearance (PASI 100) (51.1\% and $50.8 \%$ in VOYAGE 1 and $48.4 \%$ and $48.8 \%$ in VOYAGE 2 , respectively). PASI responses through both Week 100 and Week 156 were similar for adalimumab-treated patients who crossed over to receive guselkumab and patients in the guselkumab group.

Clinical responses measured using the IGA also showed similar responses [40]. At Week 100 and Week 156, respectively, $83.3 \%$ and $82.1 \%$ of patients in the guselkumab group in VOYAGE 1 achieved IGA $0 / 1$, and $83.1 \%$ and $83.0 \%$ achieved IGA $0 / 1$ in VOYAGE 2, respectively. Notably, $55.6 \%$ and $53.1 \%$ of patients in VOYAGE 1 and $52.7 \%$ and $52.9 \%$ in VOYAGE 2 achieved IGA 0 at Week 100 and Week 156, respectively. Similar IGA responses were reported for guselkumab patients and patients who crossed over from adalimumab to guselkumab at both time points.

\section{Phase III trial: NAVIGATE (guselkumab vs ustekinumab)}

Significant clinical benefits were achieved by patients who crossed over to guselkumab after an inadequate response to ustekinumab in the Phase III, randomized, double-blind trial, NAVIGATE [20]. NAVIGATE consisted of a 16-week open-label period in which all patients received ustekinumab (45 mg for patients weighing $\leq 100 \mathrm{~kg}$; $90 \mathrm{mg}$ for patients weighing $>100 \mathrm{~kg}$ ) at Weeks 0 and 4, a 28week, randomized, double-blind active-treatment period, and a 16-week follow-up period. After 16 weeks of treatment with ustekinumab, patients with an IGA score of $0 / 1$ continued to receive ustekinumab, whereas those with a score $\geq 2$ (inadequate response) were randomized to guselkumab at $100 \mathrm{mg}$ at Weeks 16, 20, and q8w, or continued ustekinumab at Week 16 and q12w through Week 44 and Week 40, respectively. Placebo injections were administered to maintain blinding.

Of the 871 patients receiving open-label ustekinumab in NAVIGATE, 268 had an IGA score $\geq 2$ of whom 135 were randomized to guselkumab and 133 to ustekinumab at Week 16; 585 patients continued open-label ustekinumab. From Week 16 through Week 40, the mean number of visits at which patients had IGA $0 / 1$ and $\geq 2$-grade improvement relative to Week 16 (primary endpoint) was significantly higher in guselkumab-treated patients compared with the ustekinumab group (1.5 vs. $0.7 ; p<0.001)$. Significantly more patients receiving guselkumab achieved IGA $0 / 1$ with a $\geq 2$ improvement at Weeks 28 and 52, relative to Week 16 , than those receiving ustekinumab (31.1\% vs. $14.3 \%$ and $36.3 \%$ vs. $17.3 \%$, respectively; both $p \leq 0.001$ ). Overall, results from NAVIGATE demonstrate that switching to guselkumab is an effective strategy in patients with an inadequate response to ustekinumab [20].

In the absence of head-to-head trials between guselkumab and ustekinumab, individual patient data from randomized controlled trials were used to indirectly compare their relative efficacy in biologic-naïve and experienced patients 
with moderate-to-severe plaque psoriasis [41]. Multivariable logistic regression analyses identified significantly higher probabilities of achieving and maintaining PASI 75, 90 , and 100 responses with guselkumab than with ustekinumab at Week 16 and through 40 weeks of treatment.

\section{Phase III trial: ECLIPSE (guselkumab vs secukinumab)}

Guselkumab also demonstrated superior long-term efficacy compared with secukinumab for the treatment of moderate-to-severe plaque-type psoriasis in the Phase III, multicentre, randomized, double-blind, active-comparator ECLIPSE trial; the first head-to-head comparator trial between guselkumab and the IL-17 inhibitor, secukinumab [21]. In this trial, patients were randomized to receive guselkumab at $100 \mathrm{mg}$, administered by subcutaneous injection at Weeks 0,4 , and q8w $(n=534)$ or secukinumab administered by two subcutaneous injections at Weeks 0 , $1,2,3$, and 4 then $\mathrm{q} 4 \mathrm{w}$ thereafter $(n=514)$, both through to Week 44.

A PASI 90 response at Week 48 (primary endpoint) was achieved by $84 \%$ of patients receiving guselkumab compared with $70 \%$ of patients in the secukinumab group $(p<0.0001)$, demonstrating the superiority of guselkumab (table 3). Non-inferiority, but not superiority, of guselkumab versus secukinumab, was established for the first major secondary endpoint (proportion of patients with a PASI 75 response at Weeks 12 and 48), however, it is likely that the dropout rate impacted on the outcome of the ECLIPSE trial as missing data were imputed as non-response. Response-over-time curves showed that maximum response rates for guselkumab were achieved after six months of treatment; after achieving maximum responses, efficacy responses were stable over time for guselkumab based on PASI 90 data compared with a decline in response for secukinumab [21]. Overall, ECLIPSE has provided invaluable insight into the effectiveness and response rates of targeting IL-23p19 versus IL-17 over a treatment duration of almost one year, with superior longer-term efficacy demonstrated for guselkumab.

A post-hoc analysis of the ECLIPSE study demonstrated higher PASI 90 and PASI 100 response rates at
Week 48 for guselkumab compared with secukinumab across all baseline body weight quartiles and body mass index categories [42]. In addition, regardless of psoriasis medication history, a numerically greater proportion of patients receiving guselkumab achieved PASI 90 response at Week 48 compared with patients treated with secukinumab [43].

\section{Phase III trial: POLARIS (guselkumab vs fumaric acid esters)}

Fumaric acid esters (FAE) are frequently prescribed in Germany as first-line systemic therapies for plaque psoriasis. The recently published POLARIS trial of 119 systemic treatment-naïve patients with moderate-to-severe plaque psoriasis demonstrated superior clinical efficacy of guselkumab compared with FAE [44]. At Week 24, the proportion of patients achieving PASI 90 (primary endpoint) or PASI 100 was significantly higher in guselkumab-treated patients than those receiving FAE (81.7\% vs. $13.6 \%$ and $31.7 \%$ vs. $3.4 \%$, respectively; both $p<0.001$ ).

\section{Other Phase III trials}

Guselkumab was efficacious and safe in 192 Japanese patients with moderate-to-severe plaque-type psoriasis in a Phase III, randomized, double-blind, placebo-controlled study [45]. The Phase III study consisted of a placebocontrolled period (Weeks 0-16), a placebo cross-over and active treatment period (Weeks 16-52), and a long-term extension phase. Patients received subcutaneous injections of guselkumab at $50 \mathrm{mg}(n=65), 100 \mathrm{mg}(n=63)$, or placebo $(n=64)$ at Weeks 0,4 , and q8w thereafter; placebo recipients crossed over to receive guselkumab at $50 \mathrm{mg}$ or $100 \mathrm{mg}$ at Weeks 16, 20, and q8w. Co-primary endpoints of IGA $0 / 1(92.3 \%$ and $88.9 \%$ vs $7.8 \%)$ and PASI $90(70.8 \%$ and $69.8 \%$ vs $0 \%$ ) at Week 16 were achieved by significantly more patients treated with guselkumab at $50 \mathrm{mg}$ or $100 \mathrm{mg}$ versus placebo, respectively (all $p<0.001$ versus placebo) [45]. IGA 0/1 and PASI 90 responses at Week 16 were maintained through Week 52 for both guselkumab doses. Importantly, treatment with guselkumab was efficacious regardless of prior use of biologics, with sustained

Table 3. Primary endpoints and major secondary endpoints in ECLIPSE [21].

\begin{tabular}{|lll|}
\hline & Guselkumab $(\boldsymbol{n = 5 3 4})$ & Secukinumab $(\boldsymbol{n}=\mathbf{5 1 4})$ \\
\hline Primary endpoint & $451(84 \%)^{*} \dagger$ & $360(70 \%)$ \\
PASI 90 at wk 48 & & \\
\hline Major secondary endpoints & $452(85 \%)^{* \dagger}$ & $412(80 \%)$ \\
PASI 75 at both wk 12 and wk 48 & $477(89 \%)$ & $471(92 \%)$ \\
PASI 75 at wk 12 & $369(69 \%)$ & $391(76 \%)$ \\
PASI 90 at wk 12 & $311(58 \%)$ & $249(48 \%)$ \\
PASI 100 at wk 48 & $454(85 \%)$ & $385(75 \%)$ \\
IGA 0/1 at wk 48 & $332(62 \%)$ & $259(50 \%)$ \\
IGA 0 at wk 48 & & \\
\hline
\end{tabular}

n: number of patients; IGA: Investigator Global Assessment; PASI 75: 75\% or greater improvement from baseline in Psoriasis Area and Severity Index; PASI 90: 90\% or greater improvement from baseline in Psoriasis Area and Severity Index; PASI 100: 100\% improvement from baseline in Psoriasis Area and Severity Index; wk: week. Values are reported as $n(\%) . * p<0.0001$ for non-inferiority of guselkumab vs. secukinumab. $\dagger p<0.0001$ for superiority of guselkumab vs. secukinumab. guselkumab did not show superiority over secukinumab. 
improvements in nail and scalp psoriasis supporting the potential of guselkumab as a novel therapeutic option in Japanese patients with psoriasis.

Finally, improved treatment adherence, and consequently better longer-term outcomes, may be achieved through the use of the novel, easy-to-use, One-Press patientcontrolled injector, which allows patients to manually control the speed of the injection and has an ergonomic design to facilitate operation. In the Phase III ORION study (NCT02905331), guselkumab administered using One-Press was highly efficacious and safe in patients with moderate-to-severe plaque-type psoriasis, with patients having a favourable impression of the device [46].

\section{Efficacy for difficult-to-treat body regions}

Guselkumab is highly effective in the treatment of psoriasis in difficult-to-treat body regions including the scalp, palms, and/or soles, and fingernails [47]. In a large, pooled psoriasis data set from VOYAGE 1 and 2 studies, guselkumab was superior to adalimumab for the treatment of psoriasis of the scalp and palms and/or soles and comparable to adalimumab for the fingernails. These combined trials comprise one of the largest clinical trial databases to date regarding the evaluation of regional psoriasis ( $>1,800$ patients), as discussed below.

Of all randomized patients $(n=1,829)$, those with a score $\geq 2$ for scalp-specific IGA (ss-IGA; $n=1,512$ ), Physician's Global Assessment of the hands and/or feet (hf-PGA; $n=461$ ), and fingernail PGA (f-PGA; $n=928$ ) were included in the analysis [47]. At Week 24, near-complete or complete clearance of the scalp and the palms and/or soles with at least a 2-grade improvement from baseline was achieved by significantly more guselkumab-treated patients compared with adalimumab $(85.0 \%$ vs $68.5 \%$ for ss-IGA $0 / 1$ and $80.4 \%$ vs $60.3 \%$ for hf-PGA $0 / 1$; both $p<0.001$ ), however f-PGA responses and target Nail Psoriasis Severity Index scores were comparable between treatment groups. Guselkumab was also an effective treatment for psoriatic alopecia that manifested as a paradoxical reaction to brodalumab in a case study of a 67-year-old Japanese man [48].

\section{Quality of life}

Significantly greater improvements from baseline in HRQoL (measured by the Dermatology Life Quality Index [DLQI]) and patient-reported outcomes (measured by the Psoriasis Symptoms and Signs Diary [PSSD]) were observed across both VOYAGE trials for guselkumabtreated patients compared with placebo or adalimumab at Weeks 16, 24, and 48 (table 4) [10, 18, 19, 49]. In NAVIGATE, a significantly higher number of patients randomized to guselkumab had a DLQI score of 0 or 1 (indicating no impact of psoriasis on HRQoL) at Week 52 compared with those in the ustekinumab group $(38.8 \%$ vs. $19.0 \% ; p=0.002$ ) [20]. Similarly, a significantly greater proportion of patients in the guselkumab group had a PSSD symptom or sign score of 0 at Week 52 compared to those treated with ustekinumab $(20.3 \%$ vs. $9.5 \%$ and $9.0 \%$ vs. $3.1 \%$, respectively; both $p<0.05$ ). Importantly, greater skin clearance at Week 24 was associated with greater improvements in HRQoL based on a pooled analysis of VOYAGE 1 and 2 data [50]. A post-hoc analysis of three-year data from VOYAGE 1 also demonstrated optimal improvements in patient-reported signs and symptoms and HRQoL achieved through three years of continuous treatment with guselkumab, with approximately $75 \%$ of patients achieving and maintaining a DLQI score of 0 or 1 [51].

Guselkumab treatment has also been associated with significantly greater improvements in the symptoms of anxiety and depression in patients with psoriasis compared with placebo or adalimumab [52]. A post-hoc analysis of VOYAGE 2 data demonstrated that improvements in psoriatic skin manifestations, as determined by residual absolute PASI scores, highly correlated with improvements in HRQoL, as assessed by the DLQI, the Hospital Anxiety and Depression Scale, and the Medical Outcomes Study 36-Item Short Form [53].

\section{Therapeutic efficacy for other forms of psoriasis and other indications}

Clinical benefits of guselkumab have also been established in palmoplantar pustulosis [54, 55], PsA [45, 56], and in severe and potentially life-threatening subtypes of psoriasis that are often difficult to treat such as generalized pustular psoriasis and erythrodermic psoriasis [57]. Studies are also underway to assess guselkumab efficacy and safety in other diseases (table 5).

Guselkumab efficacy (50 mg at Weeks 0 , and 4 , then q 8 w up to Week 52) was assessed through a 52-week-observation period in Japanese patients with generalized pustular psoriasis $(n=10)$ or erythrodermic psoriasis $(n=11)$, showing clinical response after one week of treatment [57]. Clinical Global Impression scores of "very much improved", "much improved" or "minimally improved" at Week 16 were achieved by $22.2 \%, 22.2 \%$, and $33.3 \%$ of generalized pustular psoriasis patients and by $45.5 \%, 27.3 \%$, and $18.2 \%$ of erythrodermic psoriasis patients, respectively.

The therapeutic potential of guselkumab in palmoplantar pustulosis was demonstrated in Japanese patients in a Phase II proof-of-concept, randomized, placebo-controlled study $(n=49)$, which showed its superior efficacy compared with placebo [54], and in a randomized, double-blind, placebocontrolled, Phase III clinical trial $(n=159)$ [55]. In the Phase III study, guselkumab-treated patients (100-mg or 200-mg subcutaneous injection at Weeks 0,4 , and 12, and q8w) showed significant improvements from baseline to Week 16 in the least-squares mean palmoplantar pustulosis area and severity index total score compared with placebo $(p \leq 0.02$ for both doses versus placebo), with positive treatment effects maintained through Week 52 [55].

Guselkumab may also represent a promising treatment option for PsA. A Phase IIa study, which was the first to demonstrate the efficacy and safety of targeting IL-23 in PsA, suggested the involvement of IL-23 in the pathogenesis of PsA, highlighting its potential as a promising treatment target [56]. In this study, patients with active PsA and psoriasis affecting at least $3 \%$ of their body surface 


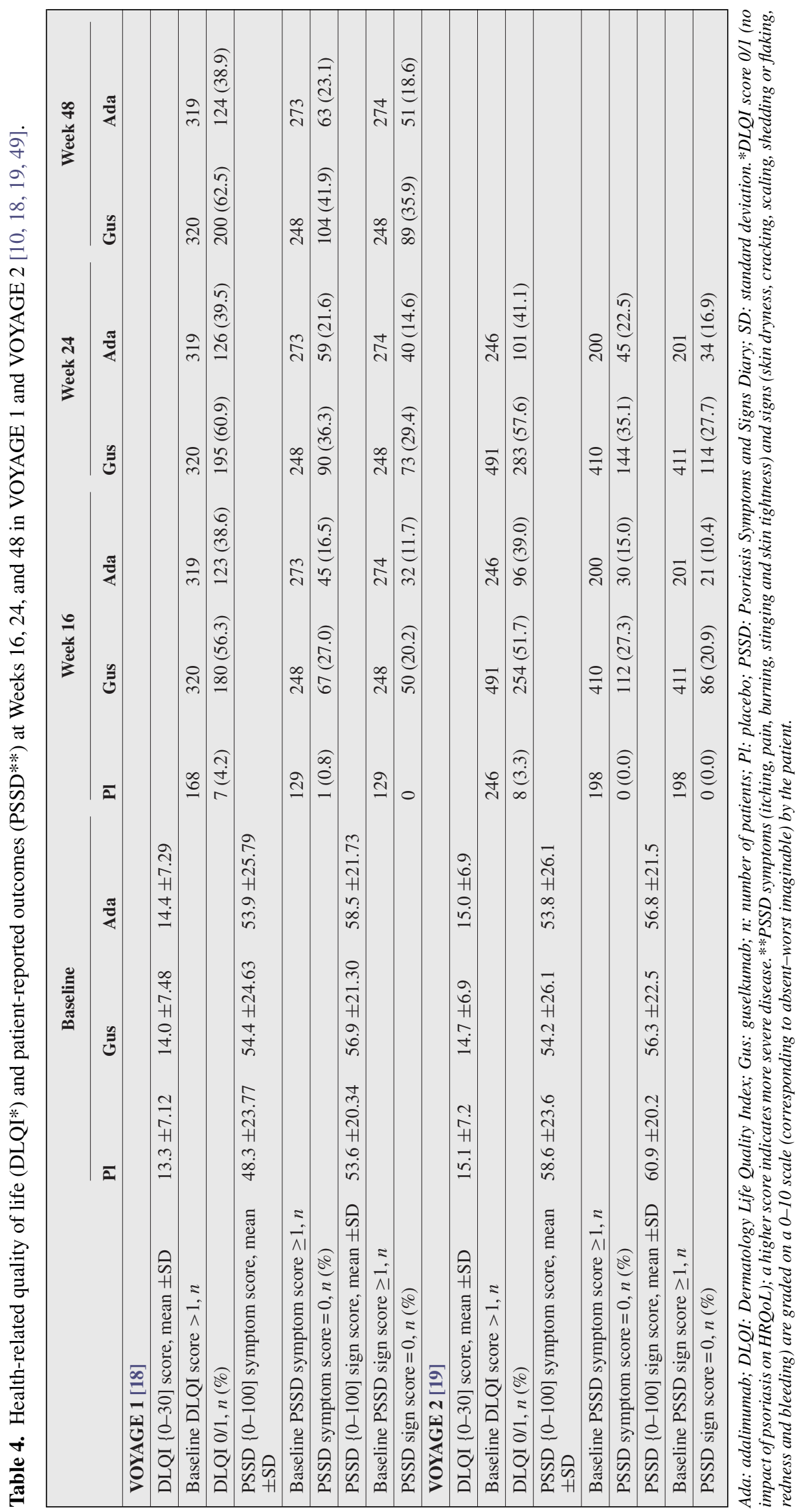




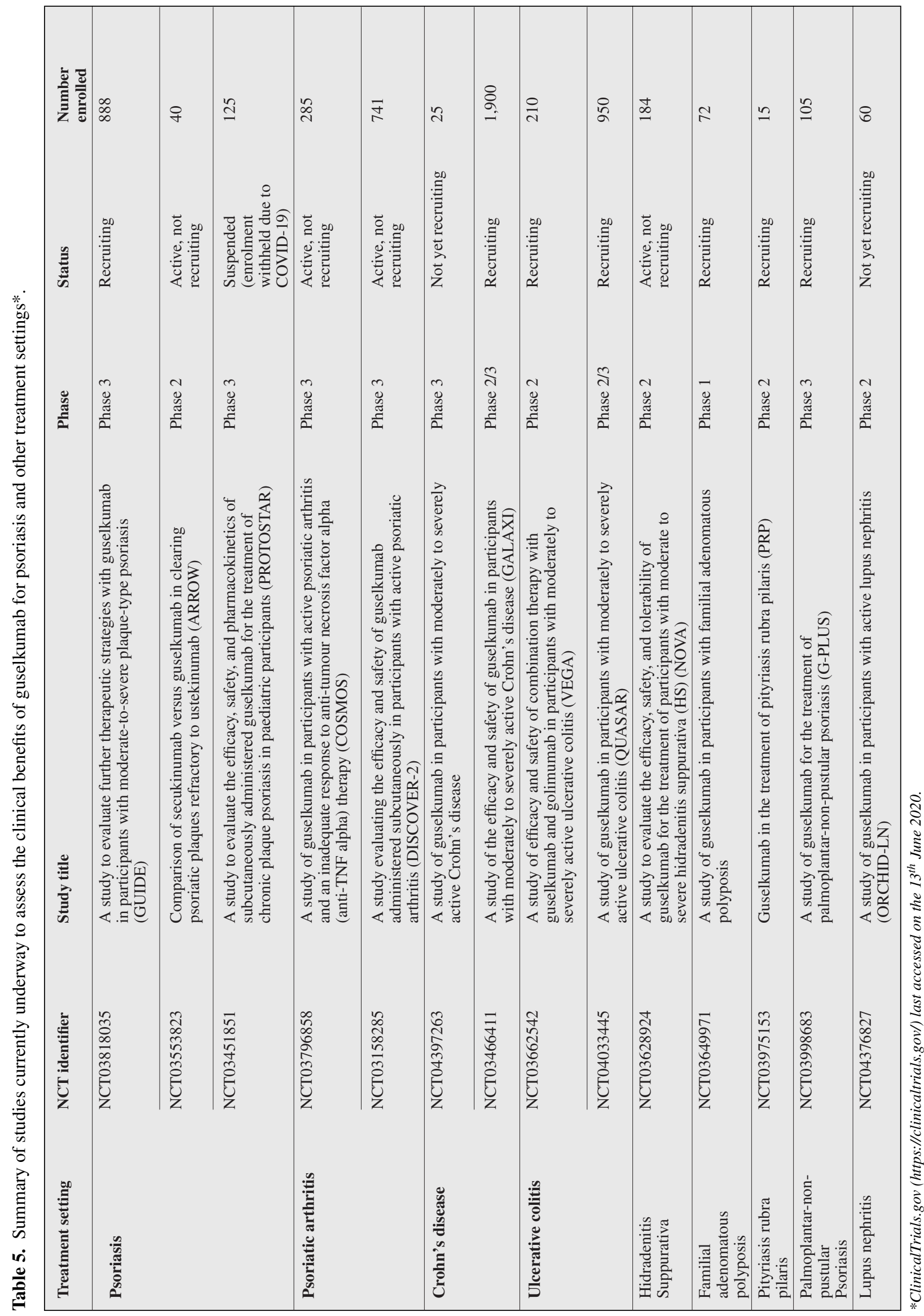


area achieved significant improvements in joint symptoms, physical function, psoriasis, enthesitis, dactylitis, and quality of life after 24 weeks of treatment with guselkumab (100 mg at Weeks 0 and 4 then q8w to Week 44, $n=100$ ) compared with placebo $(n=49)$, with efficacy maintained to Week 44 [56]. In a separate study, improvements in joint signs and symptoms associated with PsA were demonstrated at Week 16 in a small subset of Japanese patients affected by both plaque-type psoriasis and active PsA $(n=10)$ and treated with guselkumab (50 $\mathrm{mg}$ or $100 \mathrm{mg}$ ); clinical benefits were assessed at Week 16 and maintained through Week 52 [45].

Two recently published randomized, double-blind, placebocontrolled, multicentre, Phase III trials, DISCOVER-1 [58], and DISCOVER-2 [59], proved guselkumab as an effective treatment option for patients with active PsA. DISCOVER1 enrolled patients with active PsA despite treatment with standard therapies, including tumour necrosis factor inhibitors; DISCOVER-2 enrolled biologic-naive patients with active PsA. For both trials, patients were randomly assigned to receive guselkumab at $100 \mathrm{mg}$ every four weeks; guselkumab at $100 \mathrm{mg}$ at Week 0 , Week 4, and then every eight weeks, or placebo. In both trials, the primary endpoint, consisting of the proportion of patients achieving $20 \%$ or greater improvement in American College of Rheumatology response criteria (ACR20) at Week 24, was obtained by a significantly greater number of patients treated with guselkumab compared with placebo. The percentage differences for the four- and eight-week guselkumab dosing regimens versus placebo were $37 \%$ (95\% CI: 26-48) and $30 \%$ (95\% CI: 19-41), respectively, for DISCOVER-1, and $31 \%$ (95\% CI: 22-39) and 31\% (95\% CI: 23-40), respectively, for DISCOVER-2 (all $p<0.0001$ vs placebo). Signs and symptoms of PsA, as well as physical functioning and HRQoL, were significantly improved for both fourand eight-week guselkumab dosing regimens compared with placebo. Guselkumab was well-tolerated in both trials, demonstrating a favourable benefit-risk profile in patients with active PsA. In DISCOVER-2, guselkumab administered every four weeks significantly inhibited structural damage caused by disease progression in patients with active PsA at Week 24 versus placebo, thus confirming the crucial role of IL-23 in the pathogenesis of PsA.

Evidence of potential guselkumab benefits in treating Crohn's disease was described in a recent case report of a 66-year-old female with ileocolonic Crohn's disease and psoriasis [60]. Switching to guselkumab alleviated her exacerbated inflammatory bowel disease, which had occurred due to an adverse reaction to ixekizumab.

Notably, guselkumab was not effective in patients with active rheumatoid arthritis with no significant reductions in the signs and symptoms of rheumatoid arthritis in a Phase II trial [61].

\section{Real-world evidence}

There are limited real-world data related to the effectiveness, drug survival, and safety of guselkumab in clinical practice, given its recent FDA/EMA approval.

A retrospective, real-life multicentre study conducted in France and Belgium [62], evaluated guselkumab tolerability and effectiveness after 16 weeks of treatment in 180 patients with moderate-to-severe psoriasis. Overall, $38.3 \%$ and $50.6 \%$ of patients achieved PASI 100 and PASI 90 at Week 16, respectively. The proportions of patients who achieved PASI 100 at Week 16 were comparable to those observed in VOYAGE 1 (37.4\%) and VOYAGE 2 (34.1\%) trials, while the proportions of PASI 90 patients were lower (73.3\% in VOYAGE 1 and 70\% in VOYAGE 2) [18, 19]. This discrepancy could be explained by differences in the characteristics between the patient population enrolled in this study and patients in the phase III studies (i.e., disease severity, previous exposure to biologic therapies). Similar to the NAVIGATE study in which $50.4 \%$ of ustekinumabnon-responder patients achieved PASI 90 after receiving guselkumab for 16 weeks, this real-world study reported PASI 90 response in $42 \%$ (26/63) of ustekinumab-non responder patients after 16 weeks of guselkumab therapy, thus emphasizing the value of switching from anti-IL-12/23 to anti-IL-23 p19. Overall, this study confirmed the efficacy and good short-term tolerability of guselkumab for the treatment of psoriasis in the real-world setting.

Rodriguez Fernandez-Freire et al. [63] analysed 55 patients with moderate-to-severe psoriasis treated with guselkumab in another real-world study and reported significant improvements in severity indices (PASI, body surface area), pruritus visual analogue scale, and quality of life (DLQI) scores after two guselkumab injections (Week 4), with efficacy rates maintained or increased through Week 36.

Lee et al. [64] published a retrospective chart review that examined drug survival in 12 patients with psoriasis who were treated with standard dosing of guselkumab. As a comparator, 19 psoriasis patients who received ixekizumab were also included in the analyses. At the end of the study period, patients treated with guselkumab had a higher overall drug survival rate compared with patients receiving ixekizumab (91.7\% vs $73.7 \%$, respectively). Despite the small sample size, this study provides encouraging data supporting the efficacy and safety of guselkumab in real-world practice.

\section{Safety}

Guselkumab has a favourable safety profile in patients with plaque psoriasis according to results from VOYAGE 1, VOYAGE 2, NAVIGATE, and ECLIPSE [12, 18-21, 65]. Nasopharyngitis and upper respiratory tract infections were the most commonly reported adverse events (AEs) and only $1.6 \%$ to $6.7 \%$ of patients receiving guselkumab experienced at least one serious AE (SAE) (table 6) [18-21].

The types of AEs reported for the guselkumab and adalimumab treatment groups were similar during the active comparator-controlled period in VOYAGE 1 and 2 (Weeks 0-48 and Weeks 0-28, respectively) to those reported during the placebo-controlled period; the proportion of patients who experienced at least one SAE was also comparable between guselkumab and adalimumab treatment groups in both VOYAGE $1(4.9 \%$ vs $4.5 \%)$ and VOYAGE 2 $(3.6 \%$ vs $3.6 \%)$ [18, 19]. In NAVIGATE, $6.7 \%$ of patients randomized to guselkumab and $4.5 \%$ of those receiving ustekinumab had at least one SAE between Weeks 16 and 60 [20]. No additional safety concerns were raised for guselkumab in the NAVIGATE trial despite patients transitioning from ustekinumab to guselkumab without a washout period [20]. AEs in the ECLIPSE trial were gener- 
Table 6. Summary of adverse events occurring in patients with moderate-to-severe plaque psoriasis treated with guselkumab in VOYAGE 1 [18], VOYAGE 2 [19], NAVIGATE [20], and ECLIPSE [21].

\begin{tabular}{|c|c|c|c|c|c|c|c|}
\hline & \multicolumn{2}{|l|}{ VOYAGE 1} & \multicolumn{3}{|l|}{ VOYAGE 2} & \multicolumn{2}{|c|}{ NAVIGATE ECLIPSE } \\
\hline & wk 0-16 & wk $0-48$ & wk 0-16 & wk 0-28 & wk $28-48 \dagger$ & wk 16-60 & wk 0-56 \\
\hline Patients treated, $n$ & 329 & 329 & 494 & 494 & 192 & 135 & 534 \\
\hline At least $1 \mathrm{AE}$ & $170(51.7)$ & $243(73.9)$ & $235(47.6)$ & $288(58.3)$ & $99(51.6)$ & $87(64.4)$ & $416(78)$ \\
\hline \multicolumn{8}{|l|}{ Common AEs* } \\
\hline Nasopharyngitis & $30(9.1)$ & $83(25.2)$ & $35(7.1)$ & $51(10.3)$ & $22(11.5)$ & $23(17.0)$ & $118(22)$ \\
\hline Upper respiratory tract infection & 25 (7.6) & $47(14.3)$ & $16(3.2)$ & $25(5.1)$ & $9(4.7)$ & $15(11.1)$ & $83(16)$ \\
\hline Headache & $12(3.6)$ & $18(5.5)$ & $25(5.1)$ & $29(5.9)$ & $3(1.6)$ & - & $49(9)$ \\
\hline Arthralgia & $11(3.3)$ & $18(5.5)$ & - & - & - & - & $30(6)$ \\
\hline At least 1 SAE & $8(2.4)$ & $16(4.9)$ & $8(1.6)$ & $18(3.6)$ & $2(1.0)$ & $9(6.7)$ & $33(6)$ \\
\hline Infections & $85(25.8)$ & $172(52.3)$ & $106(21.5)$ & $153(31.0)$ & $55(28.6)$ & $56(41.5)$ & $313(59)$ \\
\hline Requiring treatment & $20(6.1)$ & $54(16.4)$ & $35(7.1)$ & $58(11.7)$ & $23(12.0)$ & $21(15.6)$ & $118(22)$ \\
\hline Serious infections & 0 & $2(0.6)$ & $1(0.2)$ & $3(0.6)$ & $1(0.5)$ & $1(0.7)$ & $6(1)$ \\
\hline Malignancies $\ddagger$ & 0 & $2(0.6)$ & 0 & $1(0.2)$ & 0 & $2(1.5)$ & $7(1)$ \\
\hline NMSC & $1(0.3)$ & $2(0.6)$ & 0 & $1(0.2)$ & 0 & 0 & $6(1)$ \\
\hline MACE§ & $1(0.3)$ & $1(0.3)$ & 0 & $1(0.2)$ & 0 & $2(1.5)$ & 0 \\
\hline
\end{tabular}

AE: adverse event; MACE: major adverse cardiovascular event; NMSC: non-melanoma skin cancer; SAE: serious adverse event; wk: week.Adverse events concerning the placebo group and/or active comparator group are not reported.Values are reported as $n(\%) . *$ Occurred in $\geq 5 \%$ of patients treated

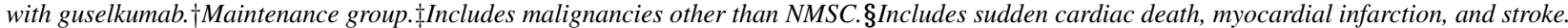

ally consistent with the known safety profile for guselkumab reported in the VOYAGE and NAVIGATE trials and current prescribing information [10,11, 18-21].

The safety profile for guselkumab remained favourable over 100 weeks of treatment in patients with moderateto-severe plaque psoriasis based on a pooled analysis of safety data from VOYAGE 1 and VOYAGE 2 trials [66]. Similarly, safety data through two years of treatment with guselkumab in VOYAGE 1 demonstrated a consistently favourable safety profile, with low and stable rates of SAEs and AEs of particular concern including serious infections, malignancy, and major adverse cardiovascular events (defined as sudden cardiac death, myocardial infarction, and stroke) [67].

Notably, the immunogenicity of guselkumab does not seem to be clinically relevant $[68,69]$. Through 100 weeks of drug exposure, only $8.5 \%$ (146 of 1,713) of guselkumab-treated patients were positive for anti-drug antibodies (ADA). Neither serum guselkumab concentration nor clinical efficacy were reduced by the development of ADA or following guselkumab withdrawal and subsequent retreatment; there was also no increase in the occurrence of injection-site reactions. These results suggest that clinical monitoring of ADA is not warranted in guselkumab-treated patients.

The development of lentigines, a rare phenomenon occurring in areas of resolving psoriatic plaques, has been reported following the treatment of psoriasis with guselkumab [70], however, these are unlikely to be specific to guselkumab as they have also been reported following treatment of psoriasis with other targeted biologic therapies [71].

\section{Conclusions}

Guselkumab, a monoclonal antibody administered subcutaneously and targeting the IL-23p19 cytokine subunit, and potentially also the IL-39p19 cytokine subunit, was the first-in-class to be approved by both the FDA and the EMA for the treatment of moderate-to-severe plaque psoriasis in adult patients who are eligible for systemic therapy with biologic drugs. The clinical development program for guselkumab is ongoing in psoriasis and other immune-mediated inflammatory diseases, including PsA, Crohn's disease, and ulcerative colitis. Understanding the importance of the IL-23/IL-17 axis in the pathogenesis of psoriasis and identifying IL-23 as responsible for the inflammatory cascade underlying its development has supported the role of IL-23 as an ideal therapeutic target.

Guselkumab is of benefit for the treatment of patients with plaque psoriasis due to its mechanism of action, its selective inhibition of IL-23-mediated cytokine activation, and its favourable pharmacokinetic properties. A further potential benefit of IL-23 inhibition is highlighted by its low dosing frequency, rapid onset of action, and long-term maintenance of response alongside its excellent safety profile. Treatment with guselkumab has demonstrated greater efficacy compared with adalimumab, ustekinumab, and secukinumab in patients with plaque psoriasis, and improved HRQoL and patient-reported symptoms and signs of psoriasis. Together these support the role of guselkumab as a valuable treatment option with durable maintenance of response over time.

Acknowledgments and disclosures. Acknowledgments: editorial support and assistance were provided by Melanie Gatt, an independent medical writer, on behalf of Springer Healthcare. This assistance was funded by Janssen. Financial support: The project was sponsored through an unconditional contribution of Janssen, without any intervention or influence in the development of the contents reported. Conflicts of interest: Andrea Chiricozzi has served as an advisory board member and consultant, and has received fees and speaker's honoraria or has participated in clinical trials for Abbvie, Almirall, Biogen, Fresenius Kabi, Leo Pharma, Lilly, Janssen, Novartis, Pfizer, 
Sanofi Genzyme, and UCB-Pharma. Antonio Costanzo is supported by grants from the Ministry of Health (grant no. CO-2013-02356463), has served as an advisory board member and consultant, and has received fees and speaker's honoraria or has participated in clinical trials for Abbvie, Almirall, Biogen,Leo Pharma, Lilly, Janssen, Novartis, Pfizer, Sanofi Genzyme, and UCB-Pharma. Maria Concetta Fargnoli has served as an advisory board member, and has received honoraria for lectures and research grants from Almirall, Celgene, Eli Lilly, Galderma, Janssen, Leo Pharma, Medac Pharma, Mylan, Novartis, Pfizer, Roche, Sanofi, UCB, Sunpharma, and Pierre Fabre. Piergiorgio Malagoli has received fees for board participation or events from Lilly, Novartis, AbbVie, Janssen, Celgene, Leopharma, and Almirall. Stefano Piaserico has served as a consultant or speaker for Abbvie, Celgene, Pfizer, Sanofi-Genzyme, Almirall, Eli Lilly, Jannsen, Galderma, and Novartis. Paolo Amerio has served as a consultant or speaker for Abbvie, Celgene, Pfizer, Sanofi-Genzyme, Eli Lilly, and Jannsen, Galderma. Federico Bardazzi has received honoraria as a consultant or speaker for Novartis, Abbvie, Janssen-cilag, Leopharma, Sandoz, Bristol Mayers, and Almirall. Luca Bianchi has received honoraria as a speaker or consultant for Abbvie, Janssen, Almirall, Eli Lilly, Leopharma, Novartis, Sanofi, Pfizer, and UCB Pharma. Carlo Giovanni Carrera has served as a board participant or speaker for Abbvie, Lilly, Jannsen, Novartis, Celgene, Almirall, and Leopharma. Andrea Conti has served as an advisory board member and consultant, and has received fees and speaker's honoraria or has participated in clinical trials for Abbvie, Almirall, Biogen, Cellgene, Leo Pharma, Eli Lilly, Janssen Cilag, MSD, Novartis, Pfizer, and UCB. Clara De Simone has received honoraria as a speaker and consultant for Abbvie, Almirall, Biogen, Eli Lilly, Leopharma, Novartis, Janssen, Sanofi, Pfizer, and UCB Pharma. Ada Lo Schiavo has received honoraria as a speaker and consultant for Novartis, Celgene, Janssen, Eli Lilly, Genzyme, and Abbvie. Giovanna Malara has received honoraria as a speaker and consultant for Janssen, Sanofi, Abbvie, Eli Lilly, Novartis, Janssen, Sanofi, and Celgene. Maria Letizia Musumeci served as advisory board member and consultant, participated to clinical trials and received fees and speaker's honoraria from Celgene, Novartis, Eli Lilly, Janssen-Cilag, Biogen and AbbVie. Aurora Parodi has participated in clinical trials and has served as an advisory board member for Abbvie, Pfizer, Celgene, Novartis, Galderma, Lilly, LEO pharma, Almirall. Ketty Peris has received grants and personal fees from Almirall and AbbVie, during the conduct of the study, and personal fees from Biogen, Lilly, Celgene, Galderma, Leo Pharma, Novartis, Pierre Fabre, Sanofi, Sandoz, Sun Pharma, and Janssen. Francesca Prignano has been a consultant, adviser and clinical study investigator for Eli-Lilly, Abbvie, Novartis, Leo-Pharma, Abiogen-Pharma, Celgene, and JanssenCilag. Franco Rongioletti has received honoraria as a consultant or speaker for Abbvie, Lilly, Janssen, Novartis, and Sanofi Genzyme. Francesco Loconsole, Giuseppe Argenziano, Nicola Balato, Paolo Dapavo, Marina Talamonti, and Concetta Potenza have no conflicts of interest to declare.

Open Access. This article is licensed under a Creative Commons Attribution 4.0 International License, which permits use, sharing, adaptation, distribution and reproduction in any medium or format, as long as you give appropriate credit to the original author(s) and the source, provide a link to the Creative Commons licence, and indicate if changes were made. The images or other third party material in this article are included in the article's Creative Commons licence, unless indicated otherwise in a credit line to the material. If material is not included in the article's Creative Commons licence and your intended use is not permitted by statutory regulation or exceeds the permitted use, you will need to obtain permission directly from the copyright holder. To view a copy of this licence, visit http://creativecommons.org/licenses/by/4.0/.

\section{References}

1. Alexander $\mathrm{H}, \mathrm{Nestle} F O$. Pathogenesis and immunotherapy in cutaneous psoriasis: what can rheumatologists learn? Curr Opin Rheumatol 2017; 29:71-8

2. Di Meglio P, Villanova F, Nestle FO. Psoriasis. Cold Spring Harb Perspect Med 2014; 4: a015354.

3. Parisi R, Symmons DP, Griffiths CE, et al. Global epidemiology of psoriasis: a systematic review of incidence and prevalence. J Invest Dermatol 2013; 133: 377-85.

4. Ferrandiz $C$, Carrascosa JM, Boada A. A new era in the management of psoriasis? The biologics: facts and controversies. Clin Dermatol 2010; $28: 81-7$

5. Fotiadou C, Lazaridou $E$, Sotiriou $E$, loannides D. Targeting IL23 in psoriasis: current perspectives. Psoriasis (Auckl.) 2018; 8: $1-5$.

6. Woo YR, Cho DH, Park HJ. Molecular mechanisms and management of a cutaneous inflammatory disorder: psoriasis. Int J Mol Sci 2017; 18: 2684.

7. Hawkes JE, Chan TC, Krueger JG. Psoriasis pathogenesis and the development of novel targeted immune therapies. J Allergy Clin Immunol 2017; 140: 645-53.

8. Markham A. Guselkumab: First global approval. Drugs 2017; 77: 1487-92.

9. Puig L. Guselkumab for the treatment of adults with moderate to severe plaque psoriasis. Expert Rev Clin Immunol 2019; 15: 589-97.

10. European Medicines Agency. Guselkumab (tremfya)_Summary of Product Characteristics_EMA. 2017. Available at: https://www.ema. europa.eu/en/medicines/human/EPAR/tremfya. laccessed 16 October 2019).

11. FDA. TREMFYA® (guselkumab) injection, for subcutaneous use. Prescribing Information. 2017. Available at: http://www. janssenlabels.com/package-insert/product-monograph/prescribinginformation/TREMFYA-pi.pdf. (accessed 4 March 2019).

12. Megna $M$, Balato $A$, Raimondo $A$, Balato N. Guselkumab for the treatment of psoriasis. Expert Opin Biol Ther 2018; 18: 459-68.

13. Sofen H, Smith S, Matheson RT, et al. Guselkumab (an IL23-specific $\mathrm{mAb}$ ) demonstrates clinical and molecular response in patients with moderate-to-severe psoriasis. J Allergy Clin Immunol 2014; 133: 1032-40

14. Gordon KB, Duffin KC, Bissonnette R, et al. A Phase 2 trial of guselkumab versus adalimumab for plaque psoriasis. N Engl J Med 2015; 373: 136-44.

15. Hu $C$, Yao $Z$, Chen $Y$, et al. A comprehensive evaluation of exposure-response relationships in clinical trials: application to support guselkumab dose selection for patients with psoriasis. J Pharmacokinet Pharmacodyn 2018; 45: 523-35.

16. Lebwohl $M$, Langley RG, Zhu $Y$, et al. Use of dose-exposureresponse relationships in Phase 2 and Phase 3 guselkumab studies to optimize dose selection in psoriasis. J Eur Acad Dermatol Venereol 2019; 33: 2082-6. 
17. Zhuang $Y$, Calderon C, Marciniak SJ Jr., et al. Firstin-human study to assess guselkumab (anti-ll-23 mAb) pharmacokinetics/safety in healthy subjects and patients with moderate-to-severe psoriasis. Eur J Clin Pharmacol 2016; 72: 1303-10.

18. Blauvelt $A$, Papp KA, Griffiths $C E$, et al. Efficacy and safety of guselkumab, an anti-interleukin-23 monoclonal antibody, compared with adalimumab for the continuous treatment of patients with moderate to severe psoriasis: results from the Phase III, double-blinded, placebo- and active comparator-controlled VOYAGE 1 trial. J Am Acad Dermatol 2017; 76: 405-17.

19. Reich K, Armstrong AW, Foley $P$, et al. Efficacy and safety of guselkumab, an anti-interleukin-23 monoclonal antibody, compared with adalimumab for the treatment of patients with moderate to severe psoriasis with randomized withdrawal and retreatment: results from the Phase III, double-blind, placebo- and active comparator-controlled VOYAGE 2 trial. J Am Acad Dermatol 2017; 76: 418-31.

20. Langley RG, Tsai TF, Flavin $S$, et al. Efficacy and safety of guselkumab in patients with psoriasis who have an inadequate response to ustekinumab: results of the randomized, double-blind, Phase III NAVIGATE trial. Br J Dermatol 2018; 178: 114-23.

21. Reich K, Armstrong AW, Langley RG, et al. Guselkumab versus secukinumab for the treatment of moderate-to-severe psoriasis (ECLIPSE): results from a Phase 3, randomised controlled trial. Lancet 2019;394: 831-9.

22. Duvallet $E$, Semerano $L$, Assier $E$, Falgarone $G$, Boissier MC. Interleukin-23: a key cytokine in inflammatory diseases. Ann Med 2011;43: 503-11.

23. Teng MW, Bowman EP, McElwee JJ, et al. IL-12 and IL-23 cytokines: from discovery to targeted therapies for immune-mediated inflammatory diseases. Nat Med 2015; 21:719-29.

24. Oppmann B, Lesley R, Blom B, et al. Novel p19 protein engages IL-12p40 to form a cytokine, IL-23, with biological activities similar as well as distinct from IL-12. Immunity 2000; 13:715-25.

25. Parham $C$, Chirica $M$, Timans J, et al. A receptor for the heterodimeric cytokine IL-23 is composed of IL-12Rbetal and a novel cytokine receptor subunit, IL-23R. J Immunol 2002; 168: 5699-708.

26. Boutet MA, Nerviani A, Gallo Afflitto G, Pitzalis C. Role of the IL23/IL-17 axis in psoriasis and psoriatic arthritis: the clinical importance of its divergence in skin and joints. Int J Mol Sci 2018; 19:530.

27. Gaffen SL, Jain R, Garg AV, Cua DJ. The IL-23-IL-17 immune axis: from mechanisms to therapeutic testing. Nat Rev Immunol $2014 ; 14: 585-600$.

28. Chiricozzi A, Romanelli P, Volpe E, Borsellino G, Romanelli M. Scanning the immunopathogenesis of psoriasis. Int J Mol Sci 2018; 19: 179.

29. Miossec $P$, Kolls JK. Targeting IL-17 and TH17 cells in chronic inflammation. Nat Rev Drug Discov 2012; 11 : 763-76.

30. Langrish $C L$, Chen $Y$, Blumenschein WM, et al. IL-23 drives a pathogenic $T$ cell population that induces autoimmune inflammation. $J$ Exp Med 2005; 201: 233-40.

31. Cai $Y$, Shen $X$, Ding C, et al. Pivotal role of dermal IL-17-producing gammadelta $T$ cells in skin inflammation. Immunity 2011;35: 596-610.

32. Hawkes JE, Yan BY, Chan TC, Krueger JG. Discovery of the IL23 /IL-17 signaling pathway and the treatment of psoriasis. J Immunol 2018; 201:1605-13

33. Yao $Z, H u C, Z$, $\mathrm{Y}$, et al. Population Pharmacokinetic modeling of guselkumab, a human lgGl lambda monoclonal antibody targeting IL-23, in patients with moderate to severe plaque psoriasis. J Clin Pharmacol 2018; 58: 613-27.

34. Nemoto O, Hirose K, Shibata S, Li K, Kubo H. Safety and efficacy of guselkumab in Japanese patients with moderate-to-severe plaque psoriasis: a randomized, placebo-controlled, ascending-dose study. $\mathrm{Br}$ J Dermatol 2018; 178: 689-96.

35. Viswanathan HN, Chau D, Milmont CE, et al. Total skin clearance results in improvements in health-related quality of life and reduced symptom severity among patients with moderate to severe psoriasis. J Dermatolog Treat 2015; 26: 235-9.
36. Griffiths CEM, Radtke MA, Youn SW, et al. Clinical response after guselkumab treatment among adalimumab PASI 90 nonresponders: Results from the VOYAGE 1 and 2 trials. J Am Acad Dermatol 2018; 79: AB78.

37. Gordon KB, Blauvelt A, Foley P, et al. Efficacy of guselkumab in subpopulations of patients with moderate-to-severe plaque psoriasis: a pooled analysis of the Phase III VOYAGE 1 and VOYAGE 2 studies. Br J Dermatol 2018; 178: 132-9.

38. Papp K, Crowley J, Rubel D, et al. Consistency of response by weight across subgroups of patients with psoriasis treated with guselkumab: Results from the VOYAGE 1 and 2 trials. J Am Acad Dermatol 2018; 79: AB87.

39. Blauvelt A, Tyring S, Philipp S, et al. Speed of response of guselkumab compared with adalimumab for the treatment of moderate-to-severe psoriasis: Results through Week 24 from the Phase 3 , double-blinded, placebo- and active comparator-controlled VOYAGE 1 and VOYAGE 2 trials. J Am Acad Dermatol 2018;79: AB267.

40. Reich K, Griffiths CEM, Gordon KB, et al. Maintenance of clinical response and consistent safety profile with up to 3 years of continuous treatment with guselkumab: results from the VOYAGE 1 and VOYAGE 2 trials. I Am Acad Dermatol 2020;82: 936-45

41. Diels J, Thilakarathne P, Cameron C, McElligott S, Schubert A, Puig L. Adjusted treatment COMPArisons between guSelkumab and UStekinumab for treatment of moderate-to-severe plaque psoriasis: the COMPASS Analysis. Br J Dermatol 2020; 183: 276-84.

42. Armstrong $A$, Blauvelt $A$, Flavin $S$, et al. Guselkumab demonstrates greater efficacy compared to secukinumab across body weight quartiles and body mass index categories: Week 48 results from the ECLIPSE trial. 24 $4^{\text {th }}$ World Congress of Dermatology (WCD); June 10-15, 2019; Milan, Italy, P524.

43. Blauvelt $A$, Vender R, Spelman $L$, et al. Efficacy of guselkumab versus secukinumab in patients with moderate-to-severe plaque psoriasis in subgroups defined by previous psoriasis medication history: results from the ECLIPSE Study. 28 ${ }^{\text {th }}$ EADV Congress; October 9-13, 2019; Madrid, Spain, P1635.

44. Thaçi $D$, Pinter $A$, Sebastian $M$, et al. Guselkumab is superior to fumaric acid esters in patients with moderate-to-severe plaque psoriasis who are naïve to systemic treatment: Results from a randomised, active comparator-controlled phase $3 \mathrm{~b}$ trial (POLARIS). Br J Dermatol 2020; 183: 265-75.

45. Ohtsuki $M$, Kubo $H$, Morishima $H$, Goto R, Zheng R, Nakagawa $H$. Guselkumab, an anti-interleukin-23 monoclonal antibody, for the treatment of moderate to severe plaque-type psoriasis in Japanese patients: efficacy and safety results from a phase 3 , randomized, double-blind, placebo-controlled study. J Dermatol 2018;45: 1053-62.

46. Ferris $L K$, Ott $E$, Jiang J, et al. Efficacy and safety of guselkumab, administered with a novel patient-controlled injector (One-Press), for moderate-to-severe psoriasis: results from the phase 3 ORION study. $J$ Dermatolog Treat 2020; 31 : 152-9.

47. Foley P, Gordon K, Griffiths CEM, et al. Efficacy of guselkumab compared with adalimumab and placebo for psoriasis in specific body regions: a secondary analysis of 2 randomized clinical trials. JAMA Dermatol 2018; 154: 676-83.

48. Hosokawa $Y$, Hamada $T$, Ashida $H$, Ikeda M. Effective treatment with guselkumab for psoriatic alopecia as paradoxical reaction. J Dermatol 2019; 46: e302-3.

49. Papp KA, Blauvelt A, Kimball AB, et al. Patient-reported symptoms and signs of moderate-to-severe psoriasis treated with guselkumab or adalimumab: results from the randomized VOYAGE 1 trial. J Eur Acad Dermatol Venereol 2018; 32: 1515-22.

50. Armstrong AW, Reich K, Foley $P$, et al. Improvement in patient-reported outcomes (dermatology life quality index and the psoriasis symptoms and signs diary) with guselkumab in moderateto-severe plaque psoriasis: results from the Phase III VOYAGE 1 and VOYAGE 2 Studies. Am J Clin Dermatol 2019;20: $155-64$. 
51. Blauvelt $A$, Han $C$, Papp $K A$, et al. Achieving and maintaining long-term optimal improvements in patient-reported symptoms, signs, and quality of life among patients with moderate to severe psoriasis treated with guselkumab: 3-year data from VOYAGE 1 (P107). J Eur Acad Dermatol Venereol 2019; 33: 49.

52. Gordon KB, Armstrong AW, Han C, et al. Anxiety and depression in patients with moderate-to-severe psoriasis and comparison of change from baseline after treatment with guselkumab vs. adalimumab: results from the Phase 3 VOYAGE 2 study. J Eur Acad Dermatol Venereol 2018; 32: 1940-9.

53. Tyring $S$, Han $C$, You $Y$, et al. Associations between improvements in patient-reported outcomes and absolute psoriasis area and severity index score: results from VOYAGE 2 (P1 18). J Eur Acad Dermatol Venereol 2019; 33: 55.

54. Terui $T$, Kobayashi $S$, Okubo $Y$, Murakami M, Hirose K, Kubo $\mathrm{H}$. Efficacy and safety of guselkumab, an anti-interleukin 23 monoclonal antibody, for palmoplantar pustulosis: a randomized clinical trial. JAMA Dermatol 2018; 154: 309-16.

55. Terui $T$, Kobayashi $S$, Okubo $Y$, et al. Efficacy and safety of guselkumab in Japanese patients with palmoplantar pustulosis: a Phase 3 randomized clinical trial. JAMA Dermatol 2019; 155: 10.

56. Deodhar A, Gottlieb AB, Boehncke WH, et al. Efficacy and safety of guselkumab in patients with active psoriatic arthritis: a randomised, double-blind, placebo-controlled, Phase 2 study. Lancet 2018; 391: 2213-24.

57. Sano S, Kubo H, Morishima H, Goto R, Zheng R, Nakagawa H. Guselkumab, a human interleukin-23 monoclonal antibody in Japanese patients with generalized pustular psoriasis and erythrodermic psoriasis: Efficacy and safety analyses of a 52-week, Phase 3, multicenter, open-label study. J Dermatol 2018; 45: 529-39.

58. Deodhar A, Helliwell PS, Boehncke WH, et al. Guselkumab in patients with active psoriatic arthritis who were biologic-naive or had previously received TNFalpha inhibitor treatment (DISCOVER-1): a double-blind, randomised, placebo-controlled phase 3 trial. Lancet 2020; 395: $1115-25$.

59. Mease PJ, Rahman $P$, Gottlieb $A B$, et al. Guselkumab in biologic-naive patients with active psoriatic arthritis (DISCOVER-2): a double-blind, randomised, placebo-controlled Phase 3 trial. Lancet 2020; 395: $1126-36$

60. Grossberg LB. A case report of successful treatment of Crohn's disease and psoriasis with guselkumab. Inflamm Bowel Dis 2019; 25: e84.
61. Smolen JS, Agarwal SK, llivanova E, et al. A randomised Phase II study evaluating the efficacy and safety of subcutaneously administered ustekinumab and guselkumab in patients with active rheumatoid arthritis despite treatment with methotrexate. Ann Rheum Dis 2017; 76: 831-9.

62. Fougerousse AC, Ghislain PD, Reguiai $Z$, et al. Effectiveness and short-term (16-week) tolerance of guselkumab for psoriasis under reallife conditions: a retrospective multicenter study. J Eur Acad Dermatol Venereol 2020; 34: e644-6.

63. Rodriguez Fernandez-Freire L, Galan-Gutierrez M, Armario-Hita JC, Perez-Gil A, Ruiz-Villaverde R. Guselkumab: short-term effectiveness and safety in real clinical practice. Dermatol Ther e13344; 2020.

64. Lee EB, Reynolds KA, Pithadia DJ, Egeberg A, Wu JJ. Drug survival of guselkumab for psoriasis in a real-world setting: a singlecenter retrospective chart review. J Dermatolog Treat 2020;31: 342-3.

65. Galluzzo M, D'Adamio S, Campione E, Bianchi L, Talamonti $M$. A safety evaluation of guselkumab for the treatment of psoriasis. Expert Opin Drug Saf 2018; 17:741-51.

66. Reich K, Papp KA, Armstrong AW, et al. Safety of guselkumab in patients with moderate-to-severe psoriasis treated through 100 weeks: a pooled analysis from the randomised VOYAGE 1 and VOYAGE 2 studies. Br J Dermatol 2019; 180: 1039-49.

67. Griffiths CEM, Papp KA, Kimball AB, et al. Long-term efficacy of guselkumab for the treatment of moderate-to-severe psoriasis: results from the Phase 3 VOYAGE 1 trial through two years. J Drugs Dermatol 2018; 17: 826-32.

68. Zhu $Y$, Marini JC, Song $M$, et al. Immunogenicity of guselkumab is not clinically relevant in patients with moderate-to-severe plaque psoriasis. J Invest Dermatol 2019; 139: 1830-4.

69. Zhu Y, Marini JC, Wasfi $Y$, et al. Impact of withdrawal and retreatment on immunogenicity of guselkumab in patients with moderate-to-severe plaque psoriasis. J Am Acad Dermatol 2018; 79: AB161.

70. Lee EB, Reynolds KA, Pithadia DJ, Wu JJ. Appearance of lentigines in psoriasis patient treated with guselkumab. Dermatol Online J 2019; 25: 13

71. Micieli R, Alavi A. Eruptive lentiginosis in resolving psoriatic plaques. JAAD Case Rep 2018; 4: 924-9. 